\title{
Effect of additives on Pleurotus ostreatus Growth on Agar medium
}

\author{
Parisa Maftoun ${ }^{1}$, Roslinda Abd Malek ${ }^{1}$, Helmi Johari Masry ${ }^{1}$, Farid Agouillal ${ }^{2}$, \\ Avnish Pareek ${ }^{3}$, Siti Zulaiha Hanapi ${ }^{1}$, Hesham A. El Enshasy ${ }^{1,4,5}$ \\ ${ }^{1}$ Instittue of Bioproduct Development (IBD), Universiti Teknologi Malaysia (UTM), Skudai, Johor, Malaysia \\ ${ }^{2}$ Research Unit on Analysis and Technological Development in Environment (URADTE), Centre de Recherche \\ Scientifique et Technique en Analyses Physico-Chimiques (CRAPC), Tipaza, Algeria. \\ ${ }^{3}$ Department of Applied Biotechnology, Ministry of Higher Education, College of Applied Sciences, Sur, \\ Sultanate of Oman \\ ${ }^{4}$ Faculty of Chemical Engineering and Energy, Universiti Teknologi Malaysia (UTM), Skudai, Johor, Malaysia \\ ${ }^{5}$ Genetic Engineering and Biotechnology Research Institute, City of Scientific Research and Technology \\ Applications (CSAT), New Burg Al Arab, Alexandria, Egypt.
}

\begin{abstract}
Oyster mushroom (Pleurotus ostreatus) is one of the most widely grown mushrooms worldwide. This type of fungus have been grown for centuries in green houses to produce mycelium and fruiting bodies and marketed as highly nutritious food. Like many other mushrooms, oyster mushroom characterized by lower growth rate which consume long time on agar medium for inoculum preparation for spawn preparation. Therefore, design of new cultivation medium to shorten the cultivation time on solid medium is necessary to reduce time and produce inoculum with less cost. This work was focused on optimization of agar medium (using the conventional potato dextrose agar medium PDA as base medium for growth and supplemented with other components such as malt extract, yeast extract, and sodium nitrates). The results clearly demonstrate that addition of malt extract and yeast extract support dense cell growth but not fast radial growth on agar medium. Addition of sodium nitrate in addition to malt and yeast extract, increase both of radial cell growth and dense growth as well. Therefore, for fast growth of Pleurotus ostreatus on agar medium its recommended to supplement the growth medium with malt extract, yeast extract, and sodium nitrate.
\end{abstract}

Keywords: Pleurotus ostreatus, Agar medium, Growth rate, medium composition, Mushroom growth

\section{Introduction}

Mushrooms have been used since centuries in different cultures in the world as food and medicine. Nowadays, they are widely cultivated in solid state cultivation in green house and also in submerged cultivation system for the production of wide range of bioactive metabolites [1-3]. Of different types of mushrooms cultivated worldwide, strains belong to the genus Pleurotus such as P. ostreatus, P. sajor-caju, and P. florida are widely cultivated as source of food and many nutraceuticals and biotherapeutic molecules [4-7]. The main interesting features of this type of mushroom is the ability to grow under different environmental conditions, ability to grow on wide range of lignocellulosic biomasses, ease of cultivation in both solid state and submerged cultivation systems, rich of high nutritious compounds (carbohydrates, proteins, vitamins, trace elements), and ability to produce many medicinally important metabolites acting as anticancer, immunomodulator, antioxidant, anti hyperglycemic, antimicrobial, anti-inflammatory, and many other therapeutic functions [8-15] . Therefore, different studies have been carried out related to classification, cultivation, bioactive compounds isolation/characterization, and preclinical research in both in vivo and in vitro models to assess the functionality of the therapeutic molecules of Pleurotus sp [16-20]. However, like many other mushrooms, low growth rate is usually is one of the main issues which increase the production cost and thus become of high interest for many researchers to develop new medium to enhance the growth rate of mushrooms. In our previous research, we developed solid culture medium to enhance cell growth for short time inoculum preparation of Cordyceps militaris using potato dextrose agar (as based medium) supplemented with yeast extract and malt extract [21]. In this work, attempts were carried out to study the effect of different medium supplements to increase the growth rate of Pleurotus ostreatus on solid agar medium through addition of different types of growth enhancing nutrients to shorten the cultivation time of this mushroom during the stage of inoculum preparation in solid medium.

\section{Materials and Methods}

Pleurotus ostreatus (WICC-F18) obtained from Wellness Industry Culture Collection (WICC), Institute of Bioproduct Development (IBD), Unviersiti Teknologi Malaysia (UTM, Malaysia) was using in this study. This strain was preserved on potato dextrose agar medium (PDA) at the first stage for mycelium production. The inoculated agar plates were incubated at $26{ }^{\circ} \mathrm{C}$ for 14 days and stored thereafter at $4{ }^{\circ} \mathrm{C}$. This was used as master bank for further strain activation. Sub-culturing were made from this first generation culture 
on PDA agar medium and used further as working cell bank. The initial experiment for characterization of cell growth was carried out on PDA agar. After this step, medium modifications were carried out using PDA medium supplemented with malt extract (MA), yeast extract (YA), glucose (G), and Sodium nitrate (SN).

The initial preliminary experiment to investigate the growth of mycelium on agar medium was carried out using standard PDA medium without any supplementation. After 12 days cultivation, full growth on agar medium was observed. From this medium, agar disk of mushroom mycelium was transferred into the center of the petri dish using crock porer. The vegetative radial growth of mycelium was measured every 2 days. The radial growth was an average of 12 measures (4 measures from the one petri dish, and the experiment run in triplicate) Figure 1 shows the typical growth of $P$. ostreatus on agar medium during 12 days cultivation.

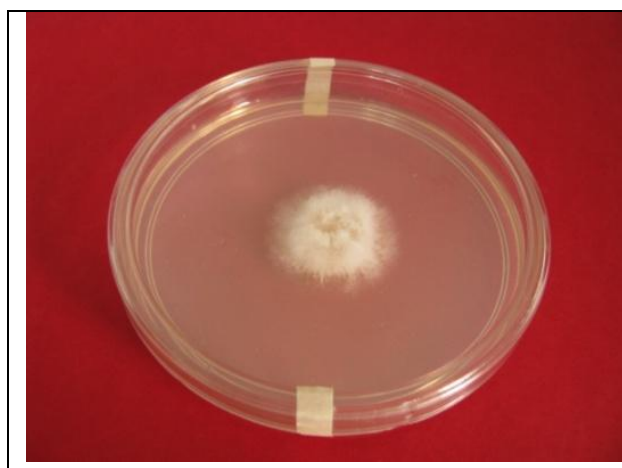

2 Days

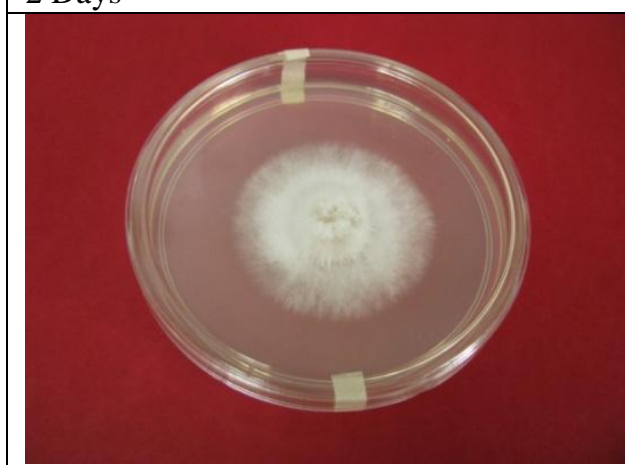

6 Days

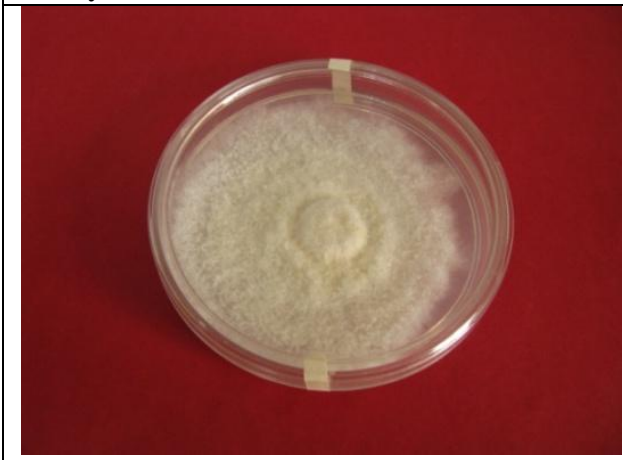

10 Days

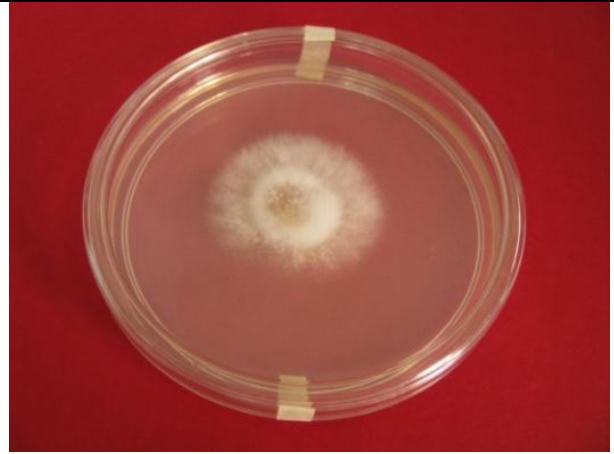

4 Days

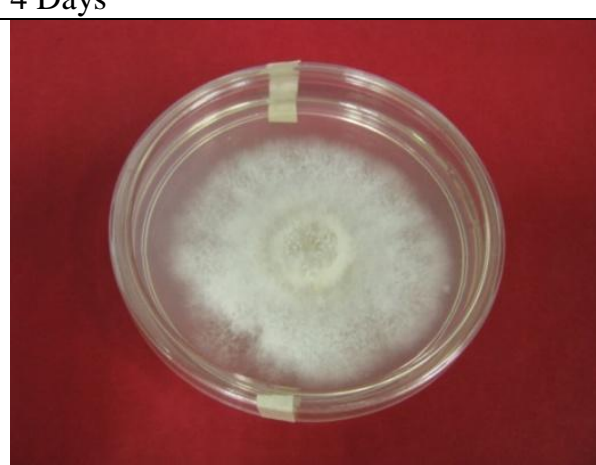

8 Days

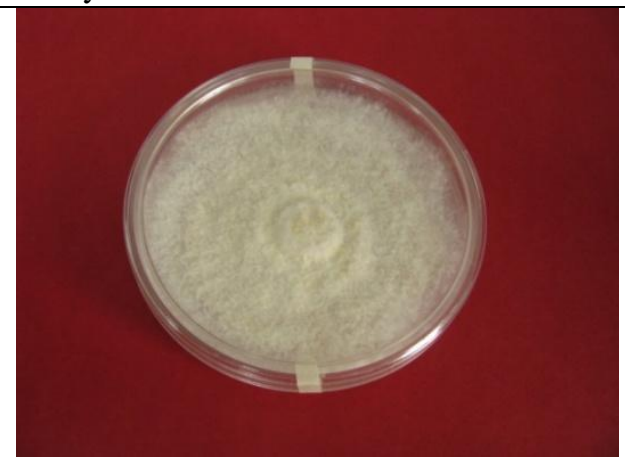

12 Days

Figure 1. A typical growth of $P$. ostreatus in mycelial form on PDA medium.

\section{Results and Discussion}

At first cultivations were carried out using standard PDA agar medium to study the growth kinetics. As shown in figure 1. In addition, cultivations were also conducted using the same methods using PDA supplemented with $5 \mathrm{~g} / \mathrm{L}$ malt extract and $2.5 \mathrm{~g} / \mathrm{L}$ yeast extract as these combined supplements provided higher growth rate in solid culture in our previous study for cultivation of Cordyceps militaris [21]. As shown in figure 2 , when following the diameter of growth on all media, no big difference was observed between PDA medium and other medium supplemented with either malt extract or yeast extract or a combination of these two nutrients. However, there was some differences in the density of mycelium growth in these modified PDA media. It have been shown that PDA plat supplemented with both malt extract and yeast extract exhibited more dense cell growth compared to other culture, whereas PDA medium without any supplementation showed the 
less dense growth on the plat (fig. 3). This could be attributed to that mycelial growth was enhanced by exogenous addition of other sugars in malt extract and nitrogen source of yeast extract.

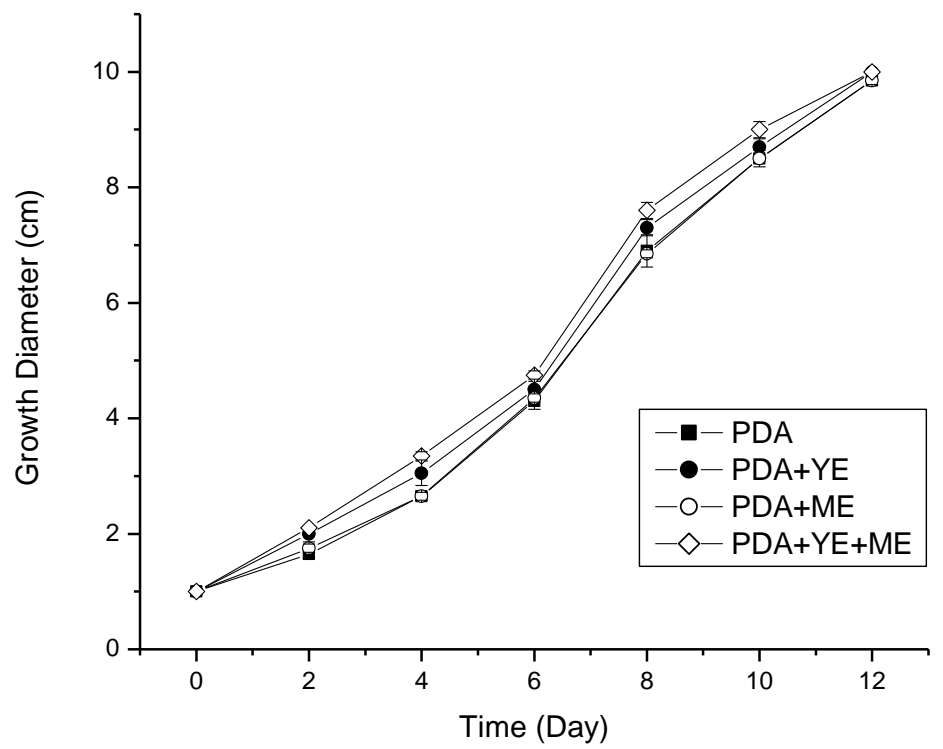

Figure 2. Change of Pleurotus ostreatus mycelial growth diameter during cultivation on agar media of different composition. PDA: Potato dextrose agar medium; PDA+YE: Potato dextrose agar medium supplemented with

$2.5 \mathrm{~g} / \mathrm{L}$ yeast extract; PDA+ME: Potato dextrose agar medium supplemented with $5 \mathrm{~g} / \mathrm{L}$ malt extract;

$\mathrm{PDA}+\mathrm{YE}+\mathrm{ME}$, Potato dextrose agar medium supplemented with $2.5 \mathrm{~g} / \mathrm{L}$ yeast extract and $5 \mathrm{~g} / \mathrm{L}$ malt extract.

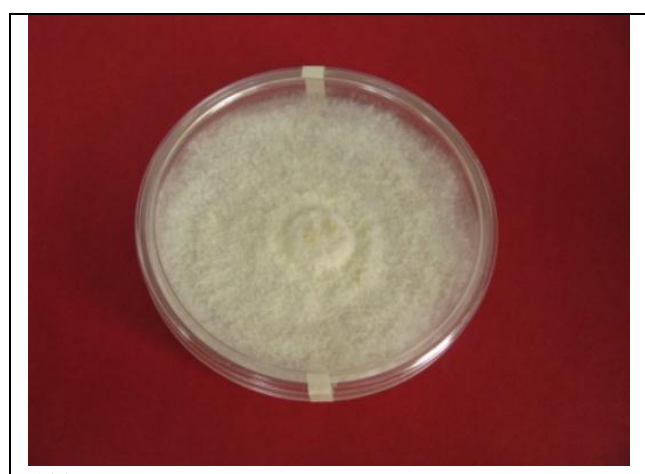

(A)

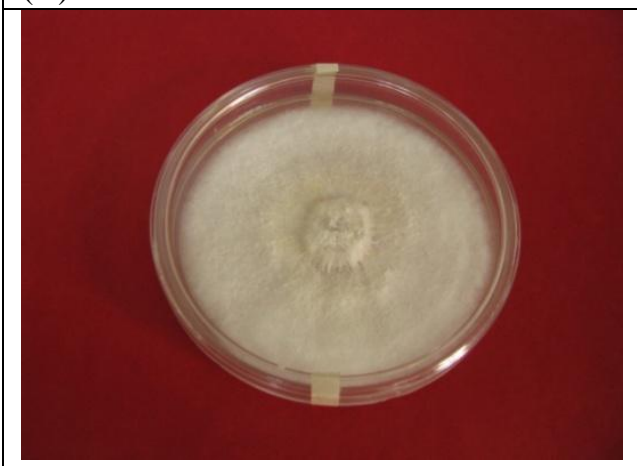

(C)

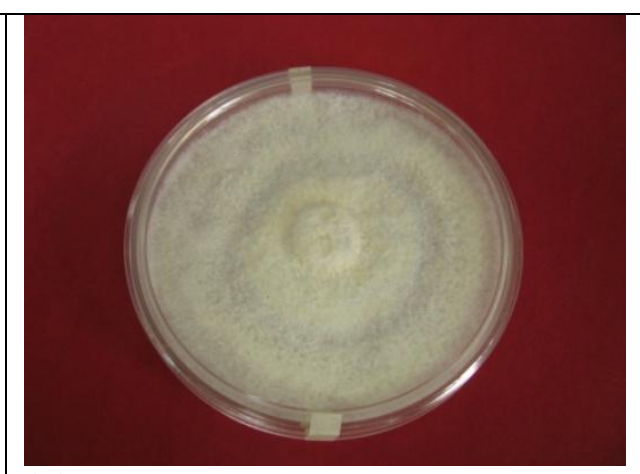

(B)

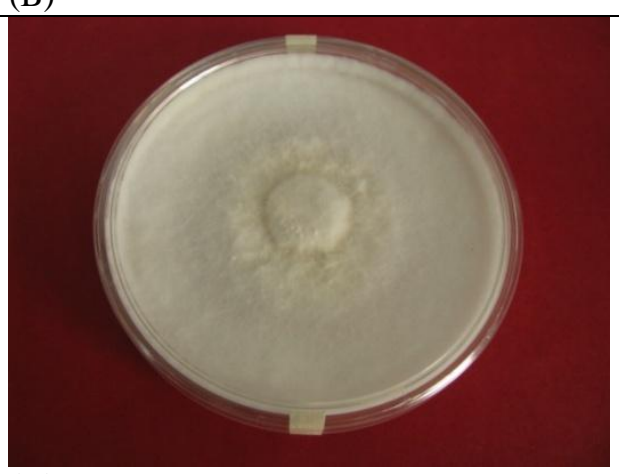

Figure 3. Macro-morphological cell growth of P. ostreatus on agar media: (A), PDA; (B), PDA+Malt extract; (C), PDA+Yeast extract; (D), PDA+Yeast extract+ Malt extract.

For further study on enhancement of radial cell growth of this mushroom, cultivations were carried out using base medium composed of (PDA+ yeast extract +malt extract) and supplemented with different 
concentrations of sodium nitrate to enhance cell growth. The preliminary experiments showed that addition $\mathrm{f}$ sodium nitrate alone without malt extract and yeast extract, didn't show significant effect on radial cell growth compared to the standard PDA medium when applied in concentration range between 0 up to $6 \mathrm{~g} / \mathrm{L}$. However, when sodium nitrate added the modified PDA medium (medium supplemented with YE and ME), it showed positive influence on the increase of radial growth rate when applied in concentration of $3 \mathrm{~g} / \mathrm{L}$, further increase beyond this concentration didn't show positive result on cell growth rate. Figure 4 shows a comparison between cell growth of standard PDA medium, PDA medium supplemented with YE and ME, PDA medium supplemented with YE, ME, and sodium nitrate. For many years, PDA is considered as standard medium for fungal cell cultivation and usually considered as the first choice for mushroom cell cultivation to support high cell growth. However, this medium is not fully optimize to enhance mushroom growth and shorten the time needed for inoculum preparation and supplementation with YE and ME showed positive impact on cell growth rate in our previous work for cultivation of $C$. militaris [21]. These based on the fact that ME is very rich source carbohydrates mixtures of different structures (maltose, sucrose, and other sugars) which support cell growth. In addition, yeast extract is well known as one of the best sources not only for nitrogen but also for vitamins, growth factors, and trace elements which are all needed for growth and production of different types of primary and secondary metabolites $[22,23]$ The results in figure 4 clearly demonstrate that addition of sodium nitrate to the cultivation medium increased the rate of radial cell growth significantly when used in concentration of $3 \mathrm{~g} / \mathrm{L}$. By addition of YE, ME, and sodium nitrate in combination, the growth diameter reached $10 \mathrm{~cm}$ after only 10 days instead of 12 days in other cultivation media. The growth was also characterized by dense mycelium as in YE and ME cultures.

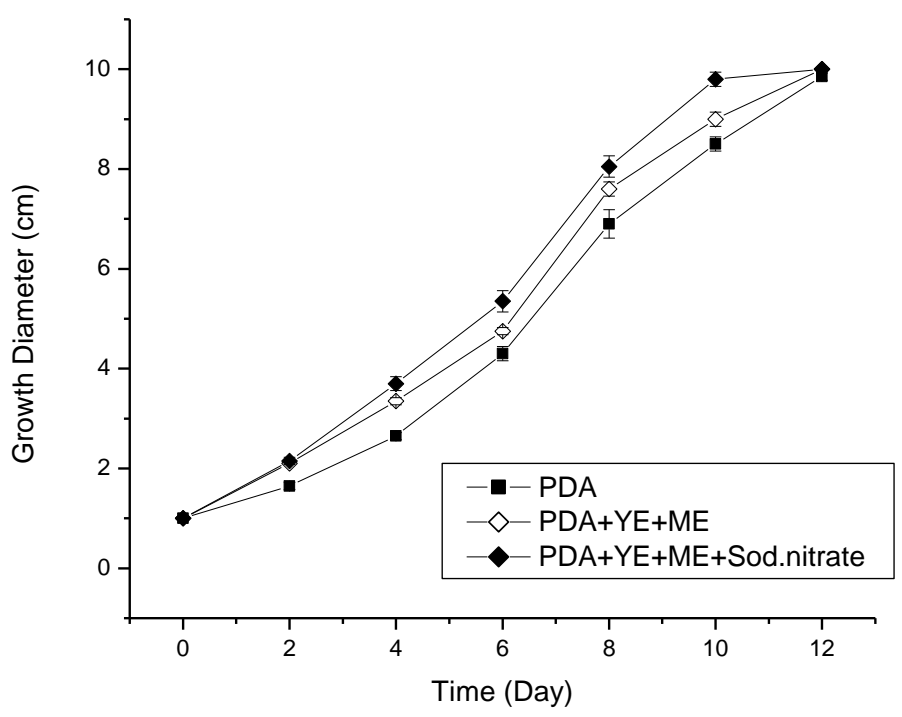

Figure 4. Radial growth of P. ostreatus when grown on standard agar medium (PDA) and PDA supplemented with yeast extract, malt extract, and sodium nitrate.

\section{Conclusion}

The obtained results in this work confirmed the supportive effects of malt extract and yeast extract for mushroom cell growth when added the traditionally used PDA for surface growth. Both of yeast extract and malt extract enhance more dense growth, whereas, addition of sodium nitrate to solid medium increase radial growth. Therefore, its recommended that during cultivation of $P$. ostreatus on solid agar medium, supplementation with yeast extract, malt extract and sodium nitrate is necessary to shorten the cultivation time and to increase the cell growth rate.

\section{Acknowledgements}

The authors would like to thank Universiti Teknologi Malaysia (UTM) for GUP grant no. Q.J130000.2509.06H98 for supporting this research. Dr. Avnish Pareek would like also to thank Dr. Ghida Banat, Department of Applied Biotechnology (CAS), Sur, Sultanate Oman, for her continuous support in RD activities 


\section{References}

[1] M Ozturk, G Tel-Cayan, A Muhammad, P Terzioglu, M Emin Duru, Mushrooms: A source of exciting bioactive. Studies in Natural Products Chemistry, 45(1), 2015, 363-456.

[2] H.A. El Enshasy, E.A. Elsayed, R. Aziz, M.A. Wadaan. Mushrooms and truffles: Historical biofactories for complementary medicine in Africa and in the Middle East. Evidence-Based Complementary and Alternative Medicine, 10, $2013: 620451$.

[3] P. Maftoun, R. Malek, M. Abbas, R. Aziz, H. El Enshasy, Bioprocess for semi-industrial production of immunomodulatory polysaccharide Pleuran by Pleurotus ostreatus in submerged culture. Journal of Scientific and Industrial Research, 72, 2013, 655662.

[4] M.A. Khan, M. Tania. Nutraceutical and medicinal importance of Pleurotus mushrooms: An overview. Food Review International, 28(1), 2012, 313-329.

[5] P Chirinang, K-O. Intarapiche., Amino acids and antioxidant properties of the oyster mushrooms, Pleurotus ostreatus and Pleurotus sajor-caju. Science Asia, 35, 2009, 326-331.

[6] E Eleftherios, M.G. Vassilis, C. Israilidis, The potential use of mushrooms $\beta$-glucans in the food industry, International Journal of Biotechnology for Wellness Industries, 3, 2014, 4-14.

[7] E.M. Mohamed, F.A. Farghaly, Bioactive compounds of fresh and dried Pleurotus ostreatus mushroom. International Journal of Biotechnology for Wellness Industries, 3, 2014, 4-14.

[8] I Lavi, D. Friesem, S. Geresh, Y. Hadar, B. Shwartz, An aqueous polysaccharide extract from the edible mushroom Pleurotus ostreatus induces anti-proliferative and pro-apototic effects on HT-29 coln cancer cells. Cancer Letter, 244(1), 2006, 61-70.

[9] M. Soltani, H. Kamyab, H.A. El Enshasy, Molecular weight (Mw) and monosaccharide composition (MC): Two major factors affecting the therapeutic action of polysaccharides extracted from Cordyceps sinensis. Journal of Pure and Appliced Microbiology, 7, 2013, 1601-1613

[10] H. El Enshasy, A. Daba, M. El Demellawy, A. Ibrahim, S. Elsayed. Bioprocess development for large scale production of anticancer exo-polysaccahride by Pleurotus ostreatus in submerged culture, Journal of Applied Science, 10, $2010,2523-2529$.

[11] H.A. El Enshasy, R. Hatti-Kaul, Mushroom immunomodulators: unique molecules with unlimited applications. Trends in Biotechnology, 31(12), 2013, 668-677.

[12] A. Saha, S. Acharya, A. Roy, Antioxidant level of wild edible mushroom: Pleurotus djamor (Fr.) Boedjin. Journal of Agriculture Technology, 8, 2012, 1343-1351.

[13] E.A. Elsayed, H.A. El Enshasy, M.A. Wadaan, R. Aziz, Mushrooms: A potential natural source of anti-inflammatory compounds for medical applications, Mediators of Inflammation, 2014, Article ID 805841, 15 pages.

[14] A.M. Younis, F-S., Wu, H.H. Shikh, Antimicrobial activity of extracts of the oyster culinary medicinal mushromm Pleurotus ostreatus (higher basidiomycetes) and identification of a new antimicrobial compound. International Journal of Medicinal Mushrooms, 17(6), 2015, 579-590.

[15] P. Maftoun, H. Johari, M. Soltani, R. Malik, N.Z. Othman, H.A. El Enshasy, The edible mushroom Pleurotus spp.: I. Biodiversity and nutritional values. International Journal of Biotechnology for Wellness Industries, 4, 2015, 67-83.

[16] H.J. Masri, P. Maftoun, R. Abd Malek, A.Z. Boumehira, A. Pareek, S.Z. Hanapi, O.M. Ling, H. El Enshasy. The edible mushroom Pleurotus spp.: II. Medicinal values. International Journal of Biotechnology for Wellness Industries, 6, $2017,1-11$.

[17] J.N. Chen, C.Y. Ma, P.F. Tsai, Y.T. Wang, J.S.B. Wu. In vitro antitumor and immunomodulatory effects of the proein PCP-3A from mushroom Pleurotus citrinoplileatus. Journal of Agriculture and Food Chemistry, 58(23), 2010, 12117-12122.

[18] E. Vamanu. In vitro antimicrobial and antioxidant activity of ethanolic extract of lyophilized mycelium of $P$. ostreatus PQMZ91109, Molecules, 2012; 217:3653-3671.

[19] C.H. Baggio, C.S. Freitas, D.F. Martins, L. Mazzardo, F.R. Smiderle, G.L. Sassaki; M. Lacomini, M.C.A. Marques, A.R.S. Santos, Antinociceptive effects of (1-3), (1-6)-linked beta-glucan isolated from Pleurotus pulmonarius in models of acute and neuropathic pain in mice: evidence for a role for glutamatergic receptors and cytokine pathways. The Journal of Pain, 11(10), $2010,965-971$.

[20] H. El Enshasy, P. Maftoun, R. Abd Malek, Pleuran: Immunomodulator polysaccharide from Pleurotus ostreatus, structure, production and application, in S. Andres, N. Baumann (Eds.) Mushrooms: Type, properties and nutrition, (New York: Nova Scientific Publisher, 2012) 153-172.

[21] M. Soltani, M. Al-Ali, N.Z. Othman, R. Malik, N. Elmarzugi, A. Aziz, H. El Enshasy, Medium composition effects on growth kinetics of Cordyceps militaris cells using agar plate method. IOSR Journal of Pharmacy and Biological Sciences, 10(1), 2015, 7982.

[22] H.A. El Enshasy, U.I. Beshay, A.I. El Diwany, H.M. Omar, A.E. El-kholy, and R. El-Najar, Improvement of rifamycins production by Amycolatopsis mediterranei in batch and fed-batch cultures. Acta Micrbiologica Polonica, 51, 2003, $301-313$.

[23] X Li, Z Li, J. Zheng, Z. Shi, L. Li, Yeast extract promotes phase shift of bio-butanol fermentation by Clostridium acetobutylicum ATCC824 using cassava as substrate. Bioresource Technolog, 125, 2012, 43-51. 